\title{
Association Between the Neutrophil Percentage-to-Albumin Ratio and Outcomes in Cardiac Intensive Care Unit Patients
}

\section{Xue Wang \\ Jie Wang (D) \\ Shujie Wu \\ Qingwei Ni \\ Peng Chen}

Department of Cardiology, The Second Affiliated Hospital and Yuying Children's Hospital, Wenzhou Medical University, Wenzhou, 325000, Zhejiang, People's

Republic of China
Correspondence: Peng Chen

Department of Cardiology, The Second Affiliated Hospital and Yuying Children's Hospital, Wenzhou Medical University, Xueyuanxi Road, No. 109, Wenzhou, 325000, Zhejiang, People's Republic of China

Emailwzcpu72@I63.com
Background: The neutrophil percentage-to-albumin ratio (NPAR) is a systemic inflammation-based predictor associated with many diseases' outcomes. Nevertheless, there are few studies on the relationship between NPAR and inflammatory markers, and more importantly, the prognostic value of NPAR in critically ill patients with cardiovascular disease (CVD) remains unknown.

Methods: The data of this retrospective cohort study were from the Medical Information Mart data for Intensive Care III database (MIMIC-III) and the Second Affiliated Hospital of Wenzhou Medical University. Linear regression, logistic regression model, and Cox regression model were used to assess the associations between NPAR levels and length of stay, renal replacement therapy (RRT) use, and 30-day, 90-day and one-year mortality, respectively. The Pearson correlation coefficient was used to present the correlation between NPAR and C-reactive protein (CRP).

Results: Our study included 1599 patients in MIMIC-III and 143 patients in the Second Affiliated Hospital of Wenzhou Medical University. The elevated NPAR was independently associated with increased 30-day, 90-day, and one-year all-cause mortality (adjusted HR, 95\% CI:1.51 (1.02-2.24); 1.61 (1.14-2.28); 1.53 (1.15-2.03); $P$ trend $=0.0297 ; 0.0053$; 0.0023 ; respectively), and it was also associated with increase the length of stay in hospital and ICU ( $\beta, 95 \%$ CI: 2.76 (1.26-4.27); 1.54 (0.62-2.47), respectively, both $P$ trend $<0.001)$. We found that patients with higher NPAR were more likely to receive RRT (OR, 95\% CI: 2.50 (1.28-4.89), $P$ trend $=0.0023)$. Moreover, we confirmed that NPAR was statistically positively correlated with CRP (correlation coefficient $\mathrm{r}=0.406, P<0.0001$ ).

Conclusion: Elevated NPAR on admission was independently associated with increased allcause mortality and length of stay among CICU patients. The results showed that CICU patients with higher NPAR were more likely to receive RRT. Besides, we also provided the evidence that there is a positive correlation between NPAR and inflammatory indicators (ie, CRP).

Keywords: neutrophil percentage-to-albumin ratio, cardiac intensive care unit, cardiovascular diseases, mortality, length of stay, renal replacement therapy, C-reactive protein

\section{Introduction}

The cardiovascular disease (CVD) is the second leading cause of mortality worldwide, accounting for 17 million deaths in 2013. ${ }^{1}$ Cardiovascular disease (CVD) is a leading cause of death and reduced quality of life, according to the latest data of the Global Burden of Disease Study, and worldwide prevalence is increasing. The cardiac intensive 
care unit (CICU) is a special care and management system for patients with severe cardiovascular disease (SCVD). SCVD has a high mortality rate, and identification of its prognostic indicators can assist medical decision-making and identification of high-risk patients. Accordingly, the effect of early biomarkers on CICU prognosis is worth exploring. ${ }^{2}$

Atherosclerosis and CVD are closely associated with inflammation. ${ }^{3}$ Neutrophils mediate early inflammatory responses, and be used to predict cardiovascular mortality. When a high neutrophil count is combined with other inflammatory markers or included in a multi-marker risk prediction model, it appears that it may provide clinically additional prognostic information. ${ }^{4}$ Most evidence suggests that changes in acute-phase proteins such as serum albumin and prealbumin do not reflect poor nutritional status but rather indicate the severity of inflammation and illness in acute disease. ${ }^{5-7}$ Recently, the neutrophil percentage-to-albumin ratio (NPAR), which neutrophil percentage are divided by serum albumin concentration, is used as a systemic inflammation-based predictor in patients with palliative pancreatic cancer, ${ }^{8}$ acute kidney injury, ${ }^{9}$ and septic shock. ${ }^{10}$ Nevertheless, the predictive value of NPAR on mortality in CICU patients is not known. Therefore, in this study, we aimed to explore the ability of NPAR to predict outcomes in CICU patients.

C-reactive protein (CRP) is a common clinical marker of inflammation, ${ }^{11}$ and its levels have been linked to patient outcomes with atherosclerotic disease, congestive heart failure, atrial fibrillation, myocarditis, aortic valve disease, and heart transplantation. ${ }^{12}$ However, as a novel systemic inflammatory marker, the relationship between NPAR and inflammatory markers remains unclear. Therefore, the secondary purpose of this study is to confirm that the NPAR is associated with CRP.

\section{Methods}

\section{Data Source}

We retrieved all data from an openly available critical care database called the Medical Information Mart for Intensive Care III (MIMIC-III, version 1.4), ${ }^{13}$ including data from 53,423 distinct hospital admissions. The data in MIMIC-III were collected from June 2001 to October 2012 at the Beth Israel Deaconess Medical Center (BIDMC, Boston, MA, USA). The data include general information (demographics, birth and death, ICU admission, and discharge information), vital signs, laboratory data, body fluid balance, reports, medications, and nursing records. The Protecting Human
Research Participants' exam was passed to gain access to the MIMIC-III database. The Massachusetts Institute of Technology (Cambridge, MA, USA) and BIDMC approved the establishment of the MIMIC-III database.

To verify the correlation between NPAR and CRP, we also collected data from CICU patients admitted to the Second Affiliated Hospital of Wenzhou Medical University from January 1, 2020, to December 30, 2020. That was approved by the ethics committee of the Second Affiliated Hospital of Wenzhou Medical University (Approval number: 2021-K-36-01). The data are anonymous, and the requirement for informed consent was therefore waived.

\section{Study Population}

The selected population must be adults (age $\geq 18$ ) admitted to CICU for the first time. Exclusion criteria were as follows: (1) CICU stay less than 48 hours; (2) absent neutrophil percentage or serum albumin concentration data during CICU stay; and (3) outlier $\geq \pm 3$ SD.

\section{Covariates}

The data were extracted from MIMIC-III by structure query language. Demographic parameters included age, sex, and race. Vital signs included temperature, heart rate, respiratory rate, systolic blood pressure (SBP), diastolic blood pressure (DBP), and percutaneous oxygen saturation. Laboratory parameters included white blood cell (WBC) counts, platelet counts, neutrophils percentage, lymphocytes percentage, red blood cell distribution width (RDW), hematocrit, hemoglobin, albumin, creatinine, glucose, anion gaComorbidities included cardiogenic shock, CAD, congestive heart failure (CHF), cardiac arrhythmia, peripheral vascular, hypertension, diabetes, renal disease, liver disease. The scoring system included sequential organ failure assessment score $(\mathrm{SOFA})^{14}$ and the simplified acute physiology score II (SAPS II). ${ }^{15}$ All laboratory parameters were collected within 48 hours after admission to the CICU.

On the other hand, to prove that NPAR was associated with CRP, data on neutrophil ratio, serum albumin and CRP were collected from CICU patients in the Second Affiliated Hospital of Wenzhou Medical University.

\section{Outcomes}

30-day all-cause mortality was the primary outcome. And the 90-day, one-year all-cause mortality, length of stay in hospital stay and ICU, and use of renal replacement therapy (RRT) in CICU patients as secondary outcomes. 


\section{Statistical Analysis}

Continuous variables were expressed as the mean \pm SD for normally distributed continuous variables and median (interquartile range) for non-normally distributed continuous variables. Differences between groups were identified using the Wilcoxon $W$-test or Kruskal-Wallis test. Categorical variables were expressed as number and percentage, and comparisons between groups were made using the chi-square test or Fisher's exact test as appropriate.

Cox regression model was used to assess the relationships between NPAR tertiles and all-cause mortality outcomes, and the first tertile group of NPAR served as the reference group. Variables with $\mathrm{P}<0.05$ in the univariate analysis and their associations with the outcomes of interest were included into the multivariate Cox proportional hazard models. In model I, covariates were only adjusted for age, sex, and race. In model II, we adjusted for age, sex, race, CAD, cardiogenic shock, congestive heart failure, cardiac arrhythmias, valvular disease, hypertension, diabetes, SOFA score, SAPS II score, serum creatinine, glucose, RDW, hematocrit, hemoglobin, WBC counts, and lymphocytes percentage. Moreover, restricted cubic spline was used to visually assess association between NPAR and the risk of the mortality. Linear regression model was used to evaluate the relationship between length of stay in hospital and NPAR adjusting for age, sex, race, serum creatinine, RDW, hematocrit, lymphocytes. Impact of NPAR level on the use of RRT in CICU patients was estimated using logistic regression model adjusting for age, sex, race, cardiogenic shock, congestive heart failure, cardiac arrhythmias, valvular disease, hypertension, diabetes, anion gap, serum creatinine, glucose, RDW, hematocrit, hemoglobin, WBC count and lymphocytes. We also used stratification analyses to examine the effect of NPAR among subgroups using various parameters and comorbidities. Receiver operating characteristic (ROC) curves were applied to test the sensitivity and specificity of NPAR. DeLong tests were applied to compare the area under the curves (AUC) for different parametric models.

The correlation between CRP and NPAR was examined using Pearson correlation. We also analyzed the correlation between CRP and neutrophil percentage and serum albumin. The correlation coefficients (r) were deemed statistically significant when the P-value was less than 0.05 .

A two-sided $\mathrm{p}<0.05$ was considered statistically significant. All statistical analyses were performed using EmpowerStats version 2.0 (http://www.empowerstats. $\underline{\mathrm{com} / \mathrm{cn} /)}$ ) and SPSS Statistic 26.

\section{Results}

\section{Patient Characteristics}

A total of 1599 patients in CICU from the MIMIC-III database were enrolled in this study (Figure 1). Patients were divided into three groups, with an equal number of cases: low-NPAR group, mid-NPAR group, and high-NPAR group (NPAR: <20.7; 20.7-24.8; and >24.8, respectively). The baseline characteristics of the eligible participants are summarized in Table 1. The subjects included 679 women and 920 men, most of whom were white. Patients with high NPAR values were more likely to be older and have higher heart rate, respiratory rate, serum creatinine, platelet counts, WBC counts, RDW, SOFA score, and SAPS II score. They also had lower SBP, DBP, hematocrit, and hemoglobin. These patients tended to have a history of cardiogenic shock, arrhythmia, peripheral vascular disease, renal failure, or liver disease. According to the selection criteria, we also identified 143 eligible CICU patients from the Second Affiliated Hospital of Wenzhou Medical University.

\section{Relationship Between the NPAR and Clinical Outcomes}

The clinical outcomes of the subjects across the tertiles of NPAR are shown in Table 2. In model I, after adjustments for age, sex, and race, patients with high NPAR had higher risk of 30-day, 90-day, and one-year all-cause mortality (HR, (95\% CI): 2.96 (2.18-4.01); 2.89 (2.21-3.79); 2.57 (2.06-3.20), respectively, $P$ trend $<0.0001)$ ) than the reference group. When examined as continuous variables in model I, each unit's higher NPAR was associated with increased 30-day (HR, 95\% CI: 1.08 (1.06-1.10); $P<0.0001)$, 90-day (HR, 95\% CI: 1.08 (1.07-1.10); $P<0.0001)$, and one-year 1.08

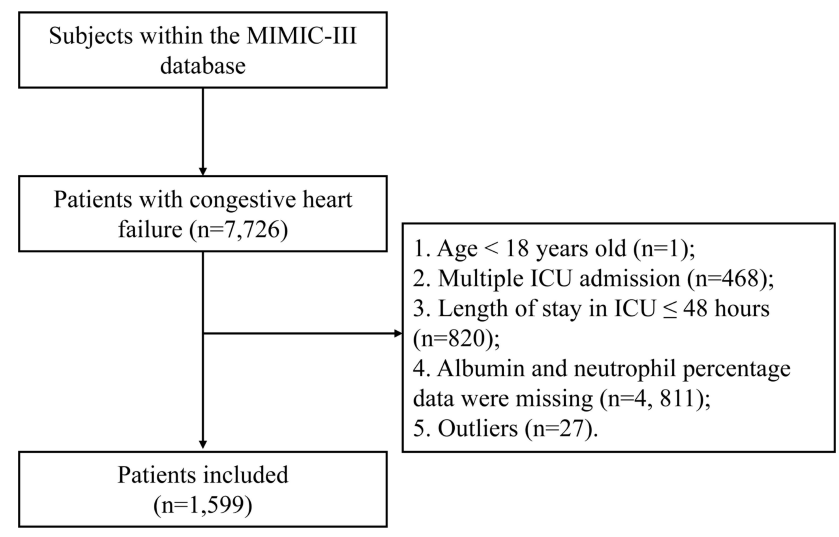

Figure I Flow chart of study population selection.

Abbreviations: ICU, intensive care unit; MIMIC-III, Medical Information Mart for Intensive Care -III. 
Table I Baseline Characteristics of the Study Population

\begin{tabular}{|c|c|c|c|c|}
\hline \multirow[t]{2}{*}{ Characteristics } & & NPAR & & \multirow[t]{2}{*}{$P$ value } \\
\hline & $<20.7$ & $20.7-24.8$ & $>24.8$ & \\
\hline NPAR & $17.46 \pm 2.88$ & $22.76 \pm 1.21$ & $29.51 \pm 4.07$ & $<0.001$ \\
\hline $\mathrm{N}$ & 533 & 533 & 533 & \\
\hline Age, years & $64.71 \pm 16.05$ & $68.43 \pm 14.89$ & $69.51 \pm 15.56$ & $<0.001$ \\
\hline $\begin{array}{l}\text { Sex, } \mathrm{n}(\%) \\
\text { Female } \\
\text { Male } \\
\text { Race, } \mathrm{n}(\%) \\
\text { Black } \\
\text { White } \\
\text { Other }\end{array}$ & $\begin{array}{l}207(38.84) \\
326(61.16) \\
72(13.51) \\
365(68.48) \\
96(18.01)\end{array}$ & $\begin{array}{l}218(40.90) \\
315(59.10) \\
40(7.50) \\
375(70.36) \\
118(22.14)\end{array}$ & $\begin{array}{c}254(47.65) \\
279(52.35) \\
36(6.75) \\
373(69.98) \\
124(23.26)\end{array}$ & $\begin{array}{r}0.010 \\
<0.001\end{array}$ \\
\hline $\begin{array}{l}\text { Vital signs } \\
\mathrm{SBP}, \mathrm{mmHg} \\
\mathrm{DBP}, \mathrm{mmHg} \\
\text { Heart rate, beats/minute } \\
\text { Respiratory rate, times/minute } \\
\mathrm{T},{ }^{\circ} \mathrm{C} \\
\mathrm{SPO}_{2}, \%\end{array}$ & $\begin{array}{c}117.37 \pm 16.67 \\
62.53 \pm 11.39 \\
80.87 \pm 16.72 \\
18.70 \pm 3.72 \\
36.80 \pm 0.58 \\
97.13 \pm 1.83\end{array}$ & $\begin{array}{r}116.16 \pm 17.25 \\
60.23 \pm 10.76 \\
81.53 \pm 15.50 \\
19.49 \pm 3.67 \\
36.74 \pm 0.68 \\
96.97 \pm 1.99\end{array}$ & $\begin{array}{l}111.89 \pm 16.82 \\
57.89 \pm 10.57 \\
87.35 \pm 17.26 \\
20.23 \pm 4.51 \\
36.81 \pm 0.73 \\
97.08 \pm 2.73\end{array}$ & $\begin{array}{l}<0.001 \\
<0.001 \\
<0.001 \\
<0.001 \\
0.183 \\
0.501\end{array}$ \\
\hline $\begin{array}{l}\text { Laboratory parameters } \\
\text { Serum albumin, g/dL } \\
\text { Serum creatinine, mg/dL } \\
\text { Glucose, mg/dL } \\
\text { RDW, \% } \\
\text { Anion gap, mmol/L } \\
\text { Hematocrit, \% } \\
\text { Hemoglobin, g/dl } \\
\text { WBC counts, } 10^{9} / \mathrm{L} \\
\text { Platelet counts, } 10^{9} / \mathrm{L} \\
\text { Neutrophils, \% } \\
\text { Lymphocytes, } \%\end{array}$ & $\begin{array}{c}3.92 \pm 0.44 \\
1.64 \pm 1.80 \\
154.19 \pm 92.04 \\
14.62 \pm 1.92 \\
16.46 \pm 4.52 \\
36.67 \pm 6.33 \\
12.34 \pm 2.19 \\
9.79 \pm 4.88 \\
242.98 \pm 107.50 \\
68.36 \pm 12.66 \\
21.93 \pm 10.74\end{array}$ & $\begin{array}{c}3.57 \pm 0.37 \\
1.89 \pm 1.77 \\
171.01 \pm 106.11 \\
14.98 \pm 2.03 \\
17.01 \pm 4.63 \\
35.21 \pm 6.35 \\
11.76 \pm 2.17 \\
12.03 \pm 5.74 \\
248.08 \pm 140.57 \\
81.07 \pm 7.94 \\
11.97 \pm 6.24\end{array}$ & $\begin{array}{c}2.93 \pm 0.42 \\
1.95 \pm 1.70 \\
163.75 \pm 88.34 \\
15.63 \pm 2.37 \\
16.53 \pm 4.63 \\
32.76 \pm 5.70 \\
10.86 \pm 1.94 \\
14.85 \pm 7.85 \\
264.86 \pm 139.03 \\
85.02 \pm 7.24 \\
8.22 \pm 5.29\end{array}$ & $\begin{array}{l}<0.001 \\
0.010 \\
0.017 \\
<0.001 \\
0.111 \\
<0.001 \\
<0.001 \\
<0.001 \\
0.077 \\
<0.001 \\
<0.001\end{array}$ \\
\hline $\begin{array}{l}\text { Comorbidities, } \mathrm{n}(\%) \\
\text { CAD } \\
\text { Cardiogenic shock } \\
\text { Congestive heart failure } \\
\text { Cardiac arrhythmias } \\
\text { Peripheral vascular disease } \\
\text { Hypertension } \\
\text { Diabetes } \\
\text { Renal failure } \\
\text { Liver disease }\end{array}$ & $\begin{array}{c}109(20.49) \\
32(6.00) \\
225(42.21) \\
186(34.90) \\
53(9.94) \\
320(60.04) \\
35(6.57) \\
106(19.89) \\
39(7.32)\end{array}$ & $\begin{array}{c}95(17.82) \\
61(11.44) \\
288(54.03) \\
191(35.83) \\
60(11.26) \\
315(59.10) \\
59(11.07) \\
131(24.58) \\
40(7.50)\end{array}$ & $\begin{array}{l}86(16.14) \\
80(15.01) \\
275(51.59) \\
226(42.40) \\
73(13.70) \\
281(52.72) \\
48(9.01) \\
137(25.70) \\
51(9.57)\end{array}$ & $\begin{array}{l}0.178 \\
<0.001 \\
<0.001 \\
0.023 \\
0.153 \\
0.032 \\
0.035 \\
0.059 \\
0.328\end{array}$ \\
\hline $\begin{array}{l}\text { Scoring systems } \\
\text { SOFA score } \\
\text { SAPS II score }\end{array}$ & $\begin{array}{c}3.80 \pm 2.77 \\
33.89 \pm 13.46\end{array}$ & $\begin{array}{c}4.40 \pm 2.85 \\
37.41 \pm 12.82\end{array}$ & $\begin{array}{c}5.51 \pm 3.43 \\
43.46 \pm 15.05\end{array}$ & $\begin{array}{l}<0.001 \\
<0.001\end{array}$ \\
\hline $\begin{array}{l}\text { Mortality, n (\%) } \\
\text { 30-day } \\
\text { 90-day } \\
\text { One year }\end{array}$ & $\begin{array}{l}57(10.69) \\
74(13.88) \\
119(22.33)\end{array}$ & $\begin{array}{l}93(17.45) \\
126(23.64) \\
180(33.77)\end{array}$ & $\begin{array}{l}166(31.14) \\
204(38.27) \\
270(50.66)\end{array}$ & $\begin{array}{l}<0.001 \\
<0.001 \\
<0.001\end{array}$ \\
\hline RRT, n (\%) & $37(6.83)$ & 47 (8.67) & $75(13.84)$ & $<0.001$ \\
\hline
\end{tabular}

Note: Data were presented as the mean \pm SD and $n(\%)$.

Abbreviations: NPAR, neutrophil percentage-albumin ratio; SBP, systolic blood pressure; DBP, diastolic blood pressure; T, temperature; SpO2, pulse oximetry-derived oxygen saturation; RDW, red blood cell distribution width; WBC, white blood cell; CAD, coronary artery disease; SOFA, sequential organ failure assessment; SAPS II, simplified acute physiology score II; RRT, renal replacement therapy. 
Table 2 HR for All-Cause Mortality Across Groups of NPAR

\begin{tabular}{|c|c|c|c|c|c|c|c|c|c|}
\hline \multirow[t]{2}{*}{ Clinical Outcomes } & \multicolumn{3}{|c|}{ Non-Adjusted } & \multicolumn{3}{|c|}{ Model I } & \multicolumn{3}{|c|}{ Model II } \\
\hline & HR (95\% Cl) & $P$ value & $P$ Trend & HR (95\% Cl) & $P$ value & $P$ Trend & HR (95\% Cl) & $P$ value & $P$ Trend \\
\hline \multicolumn{10}{|l|}{ Primary outcome } \\
\hline \multicolumn{10}{|l|}{ 30-day mortality ${ }^{8}$} \\
\hline NPAR & $1.09(1.07,1.11)$ & $<0.0001$ & & $1.08(1.06,1.10)$ & $<0.0001$ & & $1.04(1.01,1.06)$ & 0.0036 & \\
\hline NPAR tertiles & & & $<0.0001$ & & & $<0.0001$ & & & 0.0297 \\
\hline$<20.7$ & 1.0 & & & 1.0 & & & 1.0 & & \\
\hline $20.7-24.8$ & $1.70(1.22,2.37)$ & 0.0016 & & $1.53(1.10,2.14)$ & 0.0116 & & $1.20(0.82,1.75)$ & $0.34 \mathrm{II}$ & \\
\hline$>24.8$ & $3.32(2.46,4.48)$ & $<0.0001$ & & $2.96(2.18,4.01)$ & $<0.0001$ & & $1.51(1.02,2.24)$ & 0.0420 & \\
\hline \multicolumn{10}{|l|}{ Secondary outcomes } \\
\hline \multicolumn{10}{|l|}{ 90-day mortality ${ }^{\&}$} \\
\hline NPAR & $1.09(1.07,1.11)$ & $<0.0001$ & & $1.08(1.07,1.10)$ & $<0.0001$ & & $1.05(1.02,1.07)$ & $<0.0001$ & \\
\hline NPAR tertiles & & & $<0.0001$ & & & $<0.0001$ & & & 0.0053 \\
\hline$<20.7$ & 1.0 & & & 1.0 & & & 1.0 & & \\
\hline $20.7-24.8$ & $1.80(1.35,2.40)$ & $<0.0001$ & & $1.63(1.22,2.18)$ & 0.0009 & & $1.28(0.92,1.78)$ & 0.1365 & \\
\hline$>24.8$ & $3.26(2.50,4.25)$ & $<0.0001$ & & $2.89(2.21,3.79)$ & $<0.0001$ & & $1.61(1.14,2.28)$ & 0.0072 & \\
\hline \multicolumn{10}{|l|}{ One-year mortality\& } \\
\hline NPAR & $1.08(1.07,1.10)$ & $<0.0001$ & & $1.08(1.06,1.09)$ & $<0.0001$ & & $1.04(1.02,1.06)$ & $<0.0001$ & \\
\hline NPAR tertiles & & & $<0.0001$ & & & $<0.0001$ & & & 0.0023 \\
\hline$<20.7$ & 1.0 & & & 1.0 & & & 1.0 & & \\
\hline $20.7-24.8$ & $1.64(1.30,2.07)$ & $<0.0001$ & & $1.50(1.19,1.89)$ & 0.0007 & & $1.21(0.93,1.58)$ & 0.1528 & \\
\hline$>24.8$ & $2.87(2.32,3.57)$ & $<0.0001$ & & $2.57(2.06,3.20)$ & $<0.0001$ & & $1.53(1.15,2.03)$ & 0.0036 & \\
\hline \multicolumn{10}{|l|}{ Length of ICU stay ${ }^{\$}$} \\
\hline NPAR tertiles & & & $<0.0001$ & & & $<0.0001$ & & & 0.0003 \\
\hline$<20.7$ & 0 & & & 0 & & & 0 & & \\
\hline $20.7-24.8$ & $0.75(0.02,1.47)$ & 0.0431 & & $0.73(-0.00,1.45)$ & 0.0506 & & $1.32(0.70,2.52)$ & & 0.3930 \\
\hline$>24.8$ & $2.65(1.93,3.37)$ & $<0.0001$ & & $2.65(1.92,3.38)$ & $<0.0001$ & & I.54 $(0.62,2.47)$ & & 0.0011 \\
\hline \multicolumn{10}{|c|}{ Length of hospital stay ${ }^{\$}$} \\
\hline NPAR tertiles & & & $<0.0001$ & & & $<0.0001$ & & & $<0.0001$ \\
\hline$<20.7$ & 0 & & & 0 & & & 0 & & \\
\hline $20.7-24.8$ & $0.62(-0.54,1.77)$ & 0.2988 & & $0.78(-0.39,1.95)$ & 0.1903 & & $0.15(-1.16,1.46)$ & 0.8206 & \\
\hline$>24.8$ & $3.98(2.82,5.14)$ & $<0.0001$ & & $4.24(3.06,5.4 I)$ & $<0.0001$ & & $2.76(1.26,4.27)$ & 0.0003 & \\
\hline \multicolumn{10}{|l|}{$\mathrm{RRT}^{\%}$} \\
\hline NPAR tertiles & & & $<0.0001$ & & & $<0.0001$ & & & 0.0023 \\
\hline$<20.7$ & 1.0 & & & 1.0 & & & 1.0 & & \\
\hline $20.7-24.8$ & $1.30(0.83,2.03)$ & 0.2570 & & $1.42(0.90,2.25)$ & 0.1279 & & I.32 $(0.70,2.52)$ & 0.3930 & \\
\hline$>24.8$ & $2.19(1.45,3.31)$ & 0.0002 & & $2.54(1.66,3.89)$ & $<0.0001$ & & $2.50(1.28,4.89)$ & 0.0074 & \\
\hline
\end{tabular}

Notes: ${ }^{\&}$ Cox proportional hazards regression models were used to calculate hazard ratios (HR) with $95 \%$ confidence intervals (Cl). Model II was adjusted for the confounders age, sex, race, CAD, cardiogenic shock, congestive heart failure, cardiac arrhythmias, valvular disease, hypertension, diabetes, SOFA score, SAPS II score, serum

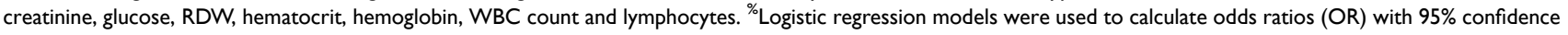
intervals (Cl). Model II was adjusted for the confounders age, sex, race, cardiogenic shock, congestive heart failure, cardiac arrhythmias, valvular disease, hypertension, diabetes, anion gap, serum creatinine, glucose, RDW, hematocrit, hemoglobin, WBC count and lymphocytes. ${ }^{\$}$ Linear regression model were used to calculate $\beta$ value with $95 \%$ confidence intervals $(\mathrm{Cl})$. Model II was adjusted for the confounders age, sex, race, serum creatinine, RDW, hematocrit, lymphocytes. Models I were adjusted for the confounders age, sex and race.

$(1.06-1.09) ; P<0.0001)$ all-cause mortality. In model II, after adjusting for more confounding factors, NPAR was also an independent predictor of 30-day, 90-day, and one-year allcause mortality of patients in CICU (high-NPAR group versus low-NPAR group: adjusted HR, (95\% CI): 1.51 (1.02-2.24); 1.61 (1.14-2.28); and 1.53 (1.15-2.03), $P$ trend $=0.0297$, 0.0053 , and 0.0023 , respectively). Likewise, when examined as continuous variables in model II, each unit's higher NPAR was still associated with increased 30-day (HR, 95\% CI: 1.04 (1.01, 1.06); $P=0.0036$ ), 90-day (HR, 95\% CI: 1.05 (1.02, 1.07); $P<0.0001$ ), and one-year (HR, 95\% CI: 1.04 (1.02, $1.06) ; P<0.0001)$ all-cause mortality independently. Besides, the restricted cubic spline also visually displayed that the $30-$ day, 90-day, and one-year all-cause mortality increased 
significantly as the NPAR increased (Figure 2 a through c). This analysis was conducted using both logarithmic transformed and untransformed data. Log RR (relative risk) can be converted to a relative risk by taking antilog. In addition, the results showed that high NPAR was associated with increased the length of stay in hospital and ICU after adjusting for multiple confounders ( $\beta, 95 \% \mathrm{CI}: 2.76$ (1.26, 4.27) 1.54 $(0.62,2.47)$, respectively, both $P$ trend $<0.0005)$ (Table 2$)$. Using the logistic regression model, after adjusting for multiple confounders, we found that patients with higher NPAR were more likely to receive RRT (OR, 95\% CI: 2.50 (1.28, 4.89), $P$ trend $=0.0023$ ) (Table 2).

We performed subgroup analysis to explore the interaction and found that none of the interactions was significant (Table 3). Moreover, the ROC curves were used to evaluate the ability of NPAR to predict 30-day all-cause mortality in CICU patients (Figure 3 ). We conducted two predictive models, and the model 1 parameter was SOFA, and the parameters of the model 2 included NPAR and SOFA. We found that the area under the curves (AUCs) for model 1 and model 2 were 0.6968 (95\% CI 0.6613-0.7323) and 0.7214 (95\% CI 0.6863-0.7564), respectively. Comparing AUCs, model 2 was a better predictive model than SOFA alone $(P=0.0181)$, and NPAR significantly enhanced the prediction efficiency of SOFA. NPAR value was an effective marker for predicting 90-day mortality in CICU patients by receiver operating characteristic curve analysis.

\section{Relationship Between the CRP and NPAR}

The results of correlation analysis between the CRP and NPAR, neutrophil percentage, and serum albumin are shown in Table 4. CRP was significantly positively correlated with NPAR and neutrophil percentage and negatively correlated with albumin. The correlation coefficients were $0.406,0.240$, and $-0.366, P<0.0001$, respectively. The correlation between NPAR and CRP was the strongest. Linear correlation analysis was performed with NPAR as Y-axis and CRP as X-axis (Figure 4). This analysis best demonstrated that NPAR is a biomarker based on systemic inflammation.

\section{Discussion}

Our results demonstrated that elevated NPAR is significantly associated with all-cause mortality in CICU patients after adjusting for several variables, the restricted cubic spline curve intuitively shows this. Although elevated NPAR was associated with increased mortality, other factors might also affect the result, including comorbid diseases; therefore, we conducted a subgroup analysis based on comorbidities and found no significant interaction, strengthening the reliability of our findings. Nevertheless, we did not elucidate the precise mechanisms underlying the association between NPAR and all-cause mortality.

Our findings can be attributed to the roles that neutrophils and serum albumin play in the innate and adaptive immune responses in inflammatory diseases such as atherosclerosis. It is worth noting that atherosclerosis, the underlying cause of the majority of CVD, is a lipiddriven, inflammatory disease of the large arteries. ${ }^{16}$ A recent European Society of Cardiology position paper concluded that inflammation is an essential regulator of atherosclerosis, dependent and independent of lipids, promoting a vicious circle of atherogenesis. ${ }^{17}$ The Canakinumab Anti-inflammatory Thrombosis Outcome Study trial demonstrated that inflammation is a critical driver of atherosclerosis and that small molecule drugs
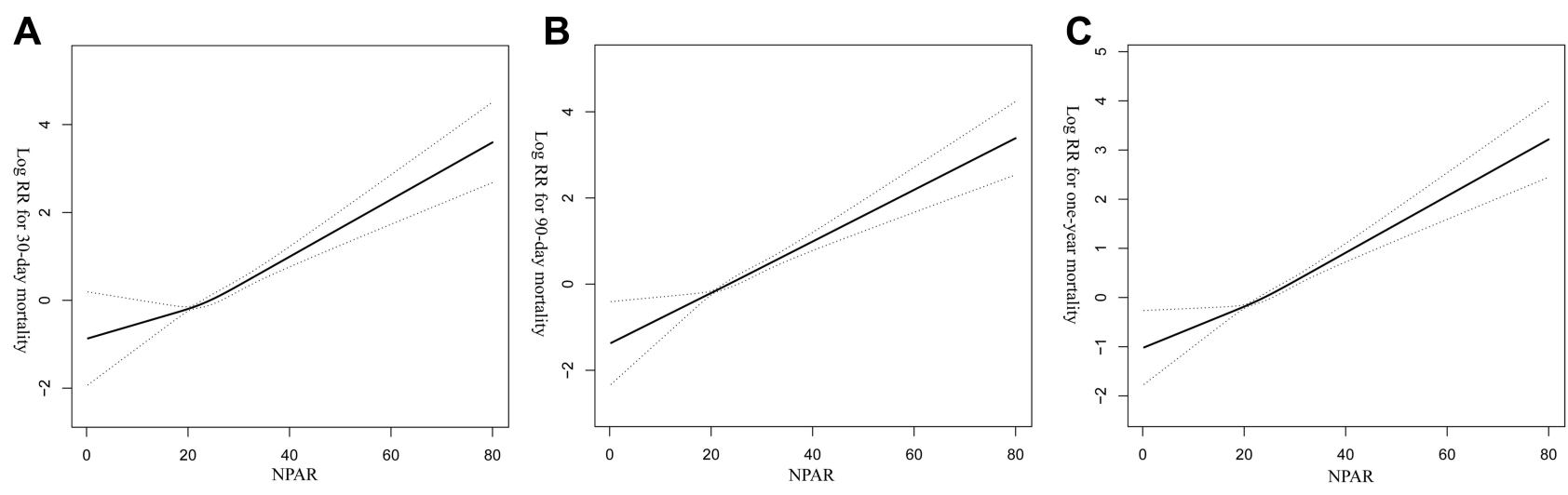

Figure 2 Restricted cubic spline demonstrates the relationship between NPAR and the risk of all-cause mortality. The resulting figures show the predicted log (relative risk) in the $y$-axis and the continuous variables in the x-axis. (A) 30-day all-cause mortality; (B) 90-day all-cause mortality; (C) one-year all-cause mortality. 
Table 3 Subgroup Analysis of the Associations Between 30-Day All-Cause Mortality and the NPAR

\begin{tabular}{|c|c|c|c|c|c|}
\hline \multirow[t]{2}{*}{ Subgroups } & \multirow[t]{2}{*}{$\mathbf{N}$} & \multirow[b]{2}{*}{$<20.7$} & \multicolumn{2}{|l|}{ NPAR } & \multirow[t]{2}{*}{$P$ for Interaction } \\
\hline & & & $20.7-24.8$ & $>24.8$ & \\
\hline CAD & & & & & 0.6361 \\
\hline No & 1308 & 1.0 & $1.50(1.04,2.18)$ & $3.04(2.17,4.28)$ & \\
\hline Yes & 290 & 1.0 & $1.82(0.85,3.90)$ & $2.65(1.28,5.49)$ & \\
\hline Cardiogenic shock & & & & & 0.5869 \\
\hline No & 1426 & 1.0 & $1.57(1.09,2.27)$ & $2.92(2.08,4.11)$ & \\
\hline Yes & 173 & 1.0 & $1.06(0.49,2.28)$ & $2.01(1.00,4.05)$ & \\
\hline Congestive heart failure & & & & & 0.2618 \\
\hline No & 811 & 1.0 & I.48 $(0.90,2.45)$ & $3.56(2.3 \mathrm{I}, 5.5 \mathrm{I})$ & \\
\hline Yes & 788 & 1.0 & $1.47(0.94,2.29)$ & $2.44(1.59,3.74)$ & \\
\hline Cardiac arrhythmias & & & & & 0.0260 \\
\hline No & 996 & 1.0 & I.I4 (0.72, I.8I) & $3.02(2.0 \mathrm{I}, 4.53)$ & \\
\hline Yes & 603 & 1.0 & $2.15(1.32,3.50)$ & $2.88(1.81,4.57)$ & \\
\hline Valvular disease & & & & & 0.2948 \\
\hline No & 1272 & 1.0 & $1.48(1.01,2.16)$ & $3.18(2.25,4.48)$ & \\
\hline Yes & 327 & 1.0 & $\mathrm{I} .67(0.85,3.3 \mathrm{I})$ & $2.26(1.16,4.40)$ & \\
\hline Peripheral vascular disease & & & & & 0.0991 \\
\hline No & 1413 & 1.0 & $1.74(1.22,2.49)$ & $3.19(2.29,4.46)$ & \\
\hline Yes & 186 & 1.0 & $0.58(0.23,1.47)$ & $1.61(0.77,3.40)$ & \\
\hline Hypertension & & & & & 0.3592 \\
\hline No & 683 & 1.0 & I.43 $(0.89,2.28)$ & $2.35(1.53,3.63)$ & \\
\hline Yes & 916 & 1.0 & $1.64(1.03,2.63)$ & $3.5 \mathrm{I}(2.28,5.4 \mathrm{I})$ & \\
\hline Diabetes & & & & & 0.6229 \\
\hline No & 1457 & 1.0 & $1.54(1.09,2.17)$ & $2.90(2.12,3.98)$ & \\
\hline Yes & 142 & 1.0 & $2.02(0.54,7.56)$ & $5.65(\mathrm{I} .5 \mathrm{I}, 2 \mathrm{I} .2 \mathrm{I})$ & \\
\hline SOFA group & & & & & 0.4270 \\
\hline$\leq 3$ & 686 & 1.0 & $1.07(0.55,2.08)$ & $\mathrm{I} .67(0.87,3.2 \mathrm{I})$ & \\
\hline$>3$ & 913 & 1.0 & $1.54(1.05,2.26)$ & $2.72(1.91,3.88)$ & \\
\hline SAPS II group & & & & & 0.8531 \\
\hline$\leq 36$ & 794 & 1.0 & I.38 $(0.69,2.76)$ & $2.60(1.34,5.04)$ & \\
\hline$>36$ & 805 & 1.0 & $1.33(0.91,1.94)$ & $2.10(1.48,2.97)$ & \\
\hline
\end{tabular}

Notes: The confounders adjustment were performed as in Model I (Table 2). Cox proportional hazards regression models were used to calculate hazard ratios (HRs) with $95 \%$ confidence intervals (Cls).

Abbreviations: NPAR, neutrophil percentage-albumin ratio; CAD, coronary artery disease; SOFA, sequential organ failure assessment; SAPS II, simplified acute physiology score II.

targeting inflammatory mediators, such as anti-interleukin $-1 \beta$ antibody, reduces the risk of CVD. ${ }^{18}$ These findings suggested that inflammation is a critical and potentially modifiable process in the development of CVD. Therefore, the novel risk predictor attempts to begin with an inflammatory perspective which may provide a novel therapeutic target.

Determining peripheral neutrophils is an inexpensive and widely available way to assess the presence of any inflammation. Previous studies showed that a higher neutrophil count was associated with a higher incidence of coronary disease, ${ }^{19}$ heart failure, ${ }^{20}$ and stroke. $^{21}$ A prospective study found that increased neutrophil count was associated with the risk of long-term death in patients with acute myocardial infarction. ${ }^{22}$ Shah, Denaxas $^{23}$ suggested that clinically recorded neutrophil count was associated with the incidence of specific CVD, even within the normal range. ${ }^{29}$ Welsh found that a higher 


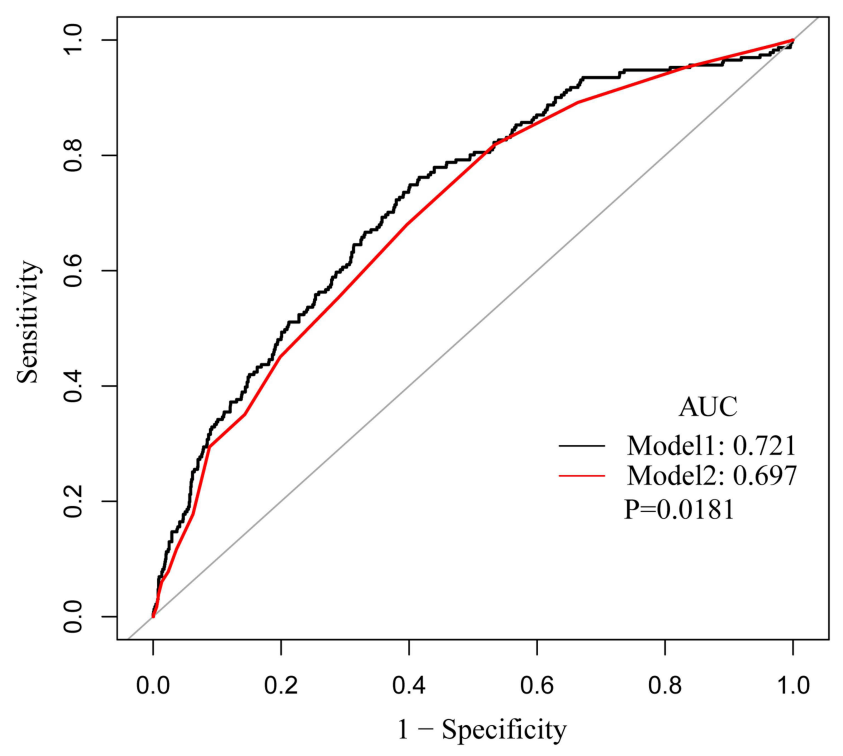

Figure 3 Receiver operating characteristic curve of NPAR value. NPAR significantly enhanced the prediction efficiency of SOFA. Model I: Parameter only include SOFA; Model 2: Parameters include SOFA and NPAR. AUC Model $I=0.6986,95 \% \mathrm{Cl}$ 0.66I3-0.7323; AUC Model 2=0.72।4, 95\% Cl 0.6863-0.7564, $\mathrm{P}=0.018 \mathrm{I}$.

Abbreviations: $\mathrm{AUC}$, area under the curve; $\mathrm{Cl}$, confidence interval.

neutrophil count is associated with a higher CVD mortality or non-fatal CVD risk, following previous data. ${ }^{23-25}$

Serum albumin (SA) is the most abundant circulating protein and possesses great antioxidant activity. ${ }^{26} \mathrm{SA}$ is the primary source of extracellular reduced sulfhydryl groups, which act as potent scavengers of reactive oxygen species, constituting the primary circulating antioxidant system. $^{27} \mathrm{SA}$ appears to inhibit the secretion of proinflammatory cytokines and complement factors (C5a) by modulating the signaling systems between inflammatory cells such as neutrophils and endothelial cells. ${ }^{28,29}$ Acute and chronic inflammatory conditions alter SA levels by regulating hepatic protein metabolism and inducing capil-

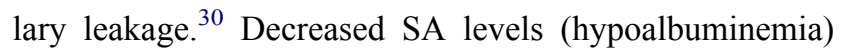
increase blood viscosity and destroy endothelial function. $^{31}$ González-Pacheco, Amezcua-Guerra ${ }^{32}$ found that low SA level was an independent predictor of newonset heart failure and in-hospital mortality in patients

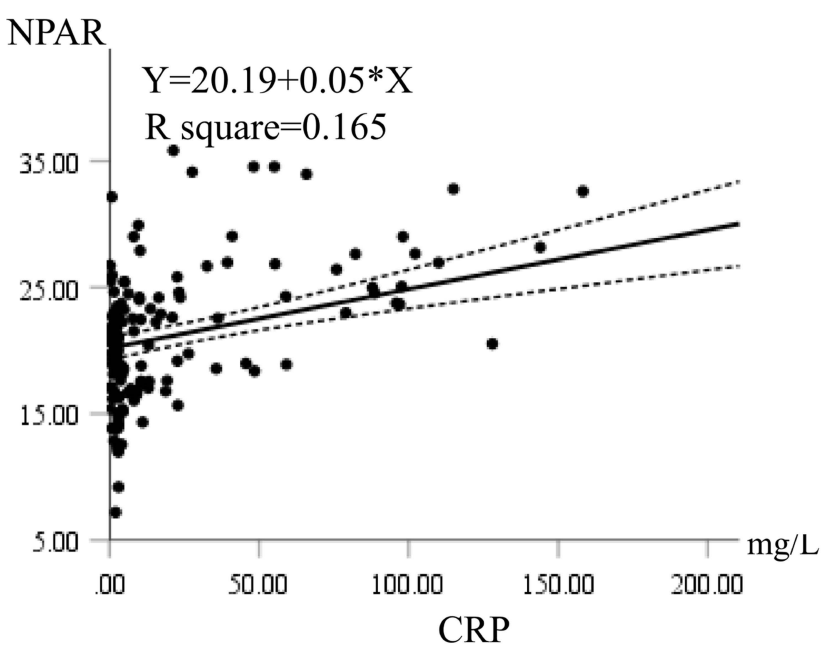

Figure 4 Correlation analysis between the NPAR and CRP. The solid line represents the appropriate value, and the dashed line represents the $95 \%$ confidence interval.

with acute cardiac syndrome. Low SA levels were associated with an increased risk of mortality and increased incidence of coronary heart disease. ${ }^{33}$

In the present study, SA levels were significantly lower in patients with higher NPAR levels. As two opposite, rapid, inexpensive, and readily available laboratory indicators, combining neutrophils percentage and serum albumin into one index could provide more accurate disease predictions. It is well known that CVD have high mortality and morbidity, especially in patients with cardiogenic shock (CS) in ICU. CS is the leading cause of hospital mortality associated with acute myocardial infarction (MI). Given the poor prognosis of critically ill patients with CS, it is necessary to find an accurate yet easily accessible prognostic predictor for risk stratification, so as to provide prognostic information and help clinical decision-making. Likewise, there is increasing evidence that the development of systemic inflammatory response syndrome (SIRS) plays an important role in both the pathogenesis of shock and the adverse outcomes of CS patients. $^{34,35}$ High levels of neutrophils in STEMI patients

Table 4 Correlation Analysis Between CRP and NPAR, Neutrophil Percentage, Albumin

\begin{tabular}{|l|c|c|c|c|}
\hline & CRP & NPAR & Serum Albumin & Neutrophil Percentage \\
\hline CRP, mg/L & $\mathrm{I}$ & & & \\
NPAR & $0.406^{* *}$ & $\mathrm{I}$ & \\
Serum albumin, g/dL & $-0.366^{* *}$ & $-0.778^{* *}$ & $\mathrm{I}$ & \\
Neutrophil percentage & $0.240^{* *}$ & $0.768^{* *}$ & $-0.264^{* *}$ & $\mathrm{I}$ \\
\hline
\end{tabular}

Note: **At 0.01 level (two tailed), the correlation was significant. 
have been found to be independently associated with an increased risk of developing late $\mathrm{CS}^{36}$ One recent study showed that hypoalbuminemia was independently associated with mortality of $\mathrm{CS}^{37}$ Accordingly, NPAR has a great potential to predict both cardiogenic shock and mortality of ICU patients.

On the other hand, our study showed that elevated NPAR was significantly related to an elevated risk of RRT use in critical ill patients with cardiovascular diseases. The acute renal replacement therapies (RRTs) are increasingly used in the cardiac intensive care unit, which are mainly used for the treatment of complications of medically refractory acute kidney injury (AKI) and cardiorenal syndrome (CRS). In addition to hemodynamic and neurohumoral abnormalities, inflammatory is an important pathophysiological mechanism of cardiorenal syndrome. ${ }^{38}$ Therefore, the relationship between NPAR and RRT indirectly reflects the inflammatory process in patients receiving RRT. It is reported that AKI was an independent prognostic factor for long-term mortality among patients with STEMI complicated by CS and treated with primary percutaneous coronary intervention (PPCI) ${ }^{39}$ There is mounting studies that have shown that the provision of RRT is associated with poor outcomes among CICU patients, with hospital mortality rates of at least $40 \%$ to $50 \%$ and 1 -year mortality $>70 \%{ }^{40,41}$ These results can partially explain that elevated NPAR was associated to significantly increase mortality and the need for RRT in CICU patients.

Moreover, many studies reported that various blood inflammatory biomarkers are associated with an increased risk of cardiovascular events. This initially sparked interest in reducing CVD risk by targeting patients with evidence of inflammation, as shown by highly sensitive CRP. ${ }^{42}$ There is evidence that serum CRP is an excellent biomarker of CVD and is an independent and robust predictor of adverse cardiovascular events. Elevated plasma levels of CRP are significantly associated with the risk of future atherothrombotic events, including stroke, coronary events, and peripheral arterial disease. ${ }^{43-46}$ It is worth noting that we have confirmed that there was a positive correlation between NAPR and CRP within this study, so it is reasonable to use NPAR as a systemic inflammatory marker. These findings suggest that the underlying inflammatory state is a major determinant of the pathophysiological mechanisms of CVD, consistent with previous studies.
This study has some advantages. To the best of our knowledge, there are no other reports on the association between NPAR and the outcomes of CICU patients. We also used a large cohort of patients, which increases the reliability of our findings. Several limitations of this study should also be noted. First, this was a single-center retrospective study, and resulting selection bias may affect the accuracy of the results. Second, we were unable to observe NPAR dynamically. The percentage of neutrophils and SA concentration used in this analysis were obtained from first blood tests after admission to the CICU. Given the dynamic nature of these indicators, bias caused by using only the first blood result cannot be avoided. Third, the more vital variables a model contains, the more accurate its prediction will be. Some critical information was not included in our model, including death from a specific cause, specific clinical symptoms, statin use, and laboratory variables (including left ventricular ejection fraction and brain natriuretic peptide). Fourth, because of the lack of neutrophils and albumin data, the sample size of this study was significantly reduced.

\section{Conclusions}

Elevated NPAR on admission was independently associated with increased of all-cause mortality and length of stay among CICU patients. The results showed that CICU patients with higher NPAR were more likely to receive RRT. Besides, we also provided the evidence that there is a positive correlation between NPAR and inflammatory indicators (ie, CRP). To validate our conclusions, prospective cohort studies are required.

\section{Funding}

There is no funding to report.

\section{Disclosure}

The authors report no conflicts of interest in this work.

\section{References}

1. Bowry ADK, Lewey J, Dugani SB, et al. The burden of cardiovascular disease in low- and middle-income countries: epidemiology and management. The Canadian Journal of Cardiology. 2015;31 (9):1151-1159. doi:10.1016/j.cjca.2015.06.028

2. Sun $H$. The neutrophil-lymphocyte ratio: a promising predictor of mortality in coronary care unit patients - a cohort study. International Immunopharmacology. 2019;74:105692. doi:10.1016/j. intimp.2019.105692

3. Steven S, Frenis K, Oelze M, et al. Vascular inflammation and oxidative stress: major triggers for cardiovascular disease. Oxid Med Cell Longev. 2019;2019:7092151. doi:10.1155/2019/7092151 
4. Hartaigh B, Bosch JA, Thomas GN, et al. Which leukocyte subsets predict cardiovascular mortality? From the LUdwigshafen RIsk and Cardiovascular Health (LURIC) Study. Atherosclerosis. 2012;224 (1):161-169. doi:10.1016/j.atherosclerosis.2012.04.012

5. Eckart A, Struja T, Kutz A, et al. Relationship of nutritional status, inflammation, and serum albumin levels during acute illness: a prospective study. The American Journal of Medicine. 2020;133 (6):713-722.e7. doi:10.1016/j.amjmed.2019.10.031

6. Jensen GL, Mirtallo J, Compher C, et al. Adult starvation and disease-related malnutrition: a proposal for etiology-based diagnosis in the clinical practice setting from the International Consensus Guideline Committee. JPEN J Parenter Enteral Nutr. 2010;34 (2):156-159. doi:10.1177/0148607110361910

7. Gehring N. Serumalbumin-a qualified parameter to determine the nutritional status? Swiss Med Wkly. 2006;136(41-42):664-669.

8. Tingle SJ, Severs GR, Goodfellow M, et al. NARCA: a novel prognostic scoring system using neutrophil-albumin ratio and Ca19-9 to predict overall survival in palliative pancreatic cancer. Journal of Surgical Oncology. 2018;118(4):680-686. doi:10.1002/jso.25209

9. Wang B. The neutrophil percentage-to-albumin ratio is associated with all-cause mortality in critically ill patients with acute kidney injury. Biomed Res Int. 2020;2020:5687672.

10. Gong Y. Increased neutrophil percentage-to-albumin ratio is associated with all-cause mortality in patients with severe sepsis or septic shock. Epidemiology and Infection. 2020;148:e87.

11. Ridker PM, Rifai N, Rose L, et al. Comparison of C-reactive protein and low-density lipoprotein cholesterol levels in the prediction of first cardiovascular events. N Engl J Med. 2002;347(20):1557-1565. doi:10.1056/NEJMoa021993

12. Osman R, L'Allier PL, Elgharib N, et al. Critical appraisal of C-reactive protein throughout the spectrum of cardiovascular disease. Vasc Health Risk Manag. 2006;2(3):221-237. doi:10.2147/ vhrm.2006.2.3.221

13. Johnson AE, Pollard TJ, Shen L, et al. MIMIC-III, a freely accessible critical care database. Sci Data. 2016;3:160035. doi:10.1038/ sdata.2016.35

14. Vincent JL, Moreno R, Takala J, et al. The SOFA (Sepsis-related Organ Failure Assessment) score to describe organ dysfunction/failure. On behalf of the Working Group on Sepsis-Related Problems of the European Society of Intensive Care Medicine. Intensive Care Med. 1996;22(7):707-710. doi:10.1007/BF01709751

15. Le Gall JR, Lemeshow S, Saulnier F. A new Simplified Acute Physiology Score (SAPS II) based on a European/North American multicenter study. JAMA. 1993;270(24):2957-2963. doi:10.1001/ jama.1993.03510240069035

16. Weber C, Noels H. Atherosclerosis: current pathogenesis and therapeutic options. Nature Medicine. 2011;17(11):1410-1422. doi: $10.1038 / \mathrm{nm} .2538$

17. Tuñón J, Badimón L, Bochaton-Piallat M-L, et al. Identifying the anti-inflammatory response to lipid lowering therapy: a position paper from the working group on atherosclerosis and vascular biology of the European Society of Cardiology. Cardiovasc Res. 2019;115(1):10-19. doi:10.1093/cvr/cvy293

18. Lutgens E, Atzler D, Döring Y, et al. Immunotherapy for cardiovascular disease. Eur Heart J. 2019;40(48):3937-3946. doi:10.1093/ eurheartj/ehz283

19. Adamsson Eryd S, Smith JG, Melander O, et al. Incidence of coronary events and case fatality rate in relation to blood lymphocyte and neutrophil counts. Arteriosclerosis, Thrombosis, and Vascular Biology. 2012;32(2):533-539. doi:10.1161/ATVBAHA.111.240416

20. Pfister R, Tuñón J, Badimón L, et al. Differential white blood cell count and incident heart failure in men and women in the EPIC-Norfolk study. Eur Heart J. 2012;33(4):523-530. doi:10.1093/eurheartj/ehr457
21. Zia E, Melander O, Björkbacka $\mathrm{H}$, et al. Total and differential leucocyte counts in relation to incidence of stroke subtypes and mortality: a prospective cohort study. Journal of Internal Medicine. 2012;272(3):298-304. doi:10.1111/j.1365-2796.2012.02526.x

22. Dragu R, Huri S, Zukermann R, et al. Predictive value of white blood cell subtypes for long-term outcome following myocardial infarction. Atherosclerosis. 2008;196(1):405-412. doi:10.1016/j. atherosclerosis.2006.11.022

23. Shah AD, Denaxas S, Nicholas O, et al. Neutrophil counts and initial presentation of 12 cardiovascular diseases: a CALIBER cohort study. $J \mathrm{Am}$ Coll Cardiol. 2017;69(9):1160-1169. doi:10.1016/j.jacc.2016.12.022

24. Wheeler J. Associations between differential leucocyte count and incident coronary heart disease: 1764 incident cases from seven prospective studies of 30,374 individuals. Eur Heart J. 2004;25 (15):1287-1292.

25. Madjid M, Fatemi O. Components of the complete blood count as risk predictors for coronary heart disease: in-depth review and update. Texas Heart Institute j. 2013;40(1):17-29.

26. Roche M. The antioxidant properties of serum albumin. FEBS Letters. 2008;582(13):1783-1787. doi:10.1016/j.febslet.2008.04.057

27. Caraceni P. Clinical indications for the albumin use: still a controversial issue. European Journal of Internal Medicine. 2013;24(8):721-728. doi:10.1016/j.ejim.2013.05.015

28. Kitano H. Role of albumin and high-density lipoprotein as endotoxin-binding proteins in rats with acute and chronic alcohol loading. Alcohol Clin Exp Res. 1996;20(1 Suppl):73A-76A.

29. Chen T-A, Tsao Y-C, Chen A, et al. Effect of intravenous albumin on endotoxin removal, cytokines, and nitric oxide production in patients with cirrhosis and spontaneous bacterial peritonitis. Scand J Gastroenterol. 2009;44(5):619-625. doi:10.1080/00365520902719273

30. Levitt DG, Levitt MD. Human serum albumin homeostasis: a new look at the roles of synthesis, catabolism, renal and gastrointestinal excretion, and the clinical value of serum albumin measurements. International Journal of General Medicine. 2016;9:229-255. doi:10.2147/IJGM.S102819

31. Joles JA, Willekes-Koolschijn N, Koomans HA. Hypoalbuminemia causes high blood viscosity by increasing red cell lysophosphatidylcholine. Kidney Int. 1997;52(3):761-770. doi:10.1038/ki.1997.393

32. González-Pacheco H, Amezcua-Guerra LM, Sandoval J, et al. Prognostic implications of serum albumin levels in patients with acute coronary syndromes. Am J Cardiol. 2017;119(7):951-958. doi:10.1016/j.amjcard.2016.11.054

33. Chan B, Dodsworth N, Woodrow J, et al. Site-specific N-terminal auto-degradation of human serum albumin. European Journal of Biochemistry. 1995;227(1-2):524-528. doi:10.1111/j.14321033.1995.tb20419.x

34. Thiele H, Allam B, Chatellier G, et al. Shock in acute myocardial infarction: the Cape Horn for trials? Eur Heart J. 2010;31 (15):1828-1835. doi:10.1093/eurheartj/ehq220

35. Selejan S-R, Pöss J, Hewera L, et al. Role of receptor for advanced glycation end products in cardiogenic shock. Crit Care Med. 2012;40 (5):1513-1522. doi:10.1097/CCM.0b013e318241e536

36. Klein A, Wiberg S, Hassager C, et al. Admission leukocyte count is associated with late cardiogenic shock development and all-cause 30-day mortality in patients with st-elevation myocardial infarction. Shock (Augusta, Ga. 2020;53(3):299-306. doi:10.1097/SHK.00000000000001369

37. Jäntti T, Tarvasmäki T, Harjola V-P, et al. Hypoalbuminemia is a frequent marker of increased mortality in cardiogenic shock. PLoS One. 2019;14(5):e0217006. doi:10.1371/journal.pone.0217006

38. Jentzer JC, Bihorac A, Brusca SB, et al. Contemporary management of severe acute kidney injury and refractory cardiorenal syndrome: JACC Council perspectives. J Am Coll Cardiol. 2020;76 (9):1084-1101. doi:10.1016/j.jacc.2020.06.070 
39. Hayıroğlu Mİ. Effect of acute kidney injury on long-term mortality in patients with ST-segment elevation myocardial infarction complicated by cardiogenic shock who underwent primary percutaneous coronary intervention in a high-volume tertiary center. Turk Kardiyoloji Dernegi Arsivi. 2020;48(1):1-9.

40. van Diepen S. Incidence, underlying conditions, and outcomes of patients receiving acute renal replacement therapies in tertiary cardiac intensive care units: an analysis from the Critical Care Cardiology Trials Network Registry. Am Heart J. 2020;222:8-14. Doi :10.1016/j. ahj.2020.01.005

41. Keleshian V, Kashani KB, Kompotiatis P, et al. Short, and long-term mortality among cardiac intensive care unit patients started on continuous renal replacement therapy. Journal of Critical Care. 2020;55:64-72. doi:10.1016/j.jcrc.2019.11.001

42. Ridker P, Danielson E, Fonseca FAH, et al. Rosuvastatin to prevent vascular events in men and women with elevated C-reactive protein. $N$ Engl J Med. 2008;359(21):2195-2207. doi:10.1056/ NEJMoa0807646
43. Wilson PWF, Pencina M, Jacques P, et al. C-reactive protein and reclassification of cardiovascular risk in the Framingham Heart Study. Circ Cardiovasc Qual Outcomes. 2008;1(2):92-97. doi:10.1161/CIRCOUTCOMES.108.831198

44. Elkind MSV, Leon V, Moon YP, et al. High-sensitivity C-reactive protein and lipoprotein-associated phospholipase A 2 stability before and after stroke and myocardial infarction. Stroke. 2009;40 (10):3233-3237. doi:10.1161/STROKEAHA.109.552802

45. Shah T, Casas JP, Cooper JA, et al. Critical appraisal of CRP measurement for the prediction of coronary heart disease events: new data and systematic review of 31 prospective cohorts. International Journal of Epidemiology. 2009;38(1):217-231. doi:10.1093/ije/ dyn 217

46. Inoue T, Kato T, Uchida T, et al. Local release of C-reactive protein from vulnerable plaque or coronary arterial wall injured by stenting. $J \mathrm{Am}$ Coll Cardiol. 2005;46(2):239-245. doi:10.1016/j.jacc.2005.04.029
International Journal of General Medicine

\section{Publish your work in this journal}

The International Journal of General Medicine is an international, peer-reviewed open-access journal that focuses on general and internal medicine, pathogenesis, epidemiology, diagnosis, monitoring and treatment protocols. The journal is characterized by the rapid reporting of reviews, original research and clinical studies
Dovepress

across all disease areas. The manuscript management system is completely online and includes a very quick and fair peer-review system, which is all easy to use. Visit http://www.dovepress.com/ testimonials.php to read real quotes from published authors. 